\title{
Efeitos ergogênicos da cafeína no teste de 3.200 metros
}

\section{Rafael Cordeiro Azevedo}

Programa de Pós-Graduação Stricto Sensu em Ciência da Motricidade Humana/PROCIMH/UCB-RJ

prof.rafael@terra.com.br

Paulo Noleto Queiroz Filho

Programa de Pós-Graduação Stricto Sensu em Ciência da Motricidade Humana/PRO$\mathrm{CIMH} / \mathrm{UCB}-\mathrm{RJ}$

cap_queiroz_exefex@yahoo.com.br

\section{Sandra Bahia Ramos}

Programa de Pós-Graduação Stricto Sensu em Ciência da Motricidade Humana/PRO. CIMH/UCB-RJ

sbr@iq.ufri.br

\section{Adalberto de Souza Rabelo}

Programa de Pós-Graduação Stricto Sensu em Ciência da Motricidade Humana/PROCIMH/UCB-RJ

as_rabelo@hotmail.com

\section{Sandro Gonzaga de Aredes}

Programa de Pós-Graduação Stricto Sensu em Ciência da Motricidade Humana/PROCIMH/UCB-RJ

sgaredes@ig.com.br

\section{Estélio Henrique Martin Dantas}

Porfessor Titular do Programa de Pós-Graduação Stricto Sensu em Ciência da Motricidade Humana da UCB-RJ

Laboratório de Biociências da Motricidade Humana - LABIMH

estelio@cobrase.com.br

AZEVEDO,R.C., FILHO, P.N.Q., RAMOS, S.B., RABELO, A.S., AREDES, S.G., DANTAS, E.H.M. Efeitos ergogênicos da cafeína no teste de 3.200 metros. Fitness \& Performance Journal, v.3, n.4, p. 225-230, 2004.

Resumo: Esta pesquisa tem como objetivo verificar os efeitos ergogênicos da cafeína em teste máximo de 3.200 metros. A amostra foi composta por 12 atletas, do sexo masculino, com idade média de $33,5 \pm 8$ anos, estatura de 176,1 $\pm 7,8 \mathrm{~cm}$, peso de $68,9 \pm 8 \mathrm{~kg}$ e tempos de treinamento de $13 \pm 7,3$ anos. Foram realizados 2 testes máximos de 3.200 metros. Após o $1^{\circ}$ teste houve um período de abstinência de cafeína e no $2^{\circ}$ houve ingestão da substância. Foi verificado que 11 dos 12 atletas obtiveram uma melhora nos tempos médios de $8,08 \pm 6,01 \mathrm{seg}$. O coeficiente de correlação do teste " $t$ " pareado apresentou uma significância de $p<0,05$. Concluímos que o uso da cafeína, após uma semana de abstinência, produz efeito ergogênico significativo na performance de atletas em teste máximo de 3.200 metros.

Palabras-chave: Recurso ergogênico, cafeína, performance.

Endereço para correspondência:

Rua Conde de Irajá, 122/402 - Botafogo - CEP 22271-020 


\section{Ergogenic Efects of caffeine in test of 3.200 meters}

The aim of the present study was to verify the ergogenic effects of caffeine over a maximum test of 3.200 meters. The sample was composed of 12 male athletes, with average age of $33,5 \pm 8$ years, height of $176,1 \pm 7,8 \mathrm{~cm}$, weight of 68,9 $\pm 8 \mathrm{~kg}$ and training time of $13 \pm 7,3$ years. Two maximum tests were performed during 3.200 meters. After the first test, there was caffeine abstinence and on the second, there was caffeine ingestion. It was observed that 11 out of the 12 athletes obtained an improvement in the average times of $8,08 \pm 6,01 \mathrm{sec}$. The correlation coefficient of the paired " $t$ " test presented a significance of $p<0,05$. In conclusion, the use caffeine after a week of abstinence, produce significant ergogenic efect in the athletes' performance in a maximum test of 3.200 meters.

Keywords: Ergogenic resource, caffeine, performance.

\section{Efectos ergogénicos de la cafeína en el test de 3.200 metros}

Esta investigación tiene como objetivo verificar los efectos ergogénicos de la cafeína en un test máximo de 3.200 metros. La muestra fue componpuesto de 12 atletas, hombres, con edad media de 33,5 \pm años, estatura de $176,1 \pm 7,8$ $\mathrm{cm}$, peso de $68,9 \pm 8 \mathrm{~kg}$ y tiempos de entrenamiento de $13 \pm 7,3$ anos. Fueron realizados 2 tests máximos de 3.200 metros. Después del $1^{\circ}$ test, se realizó una abstinencia de cafeína y en el $2^{\circ}$ una ingestión. Fue verificado que 11 de los 12 atletas obtuvieron una mejoría en los tiempos medios de $8,08 \pm 6,01$ seg. El coeficiente de correlación del test " $\mathrm{t}$ " pareado apresentó una significatividad de $p<0,05$. Se puede concluir que el uso de la cafeína, después de una semana de abstinencia, produce efecto ergogénico significativo en la performance de atletas en un test máximo de 3.200 metros.

Palabras clave - Recurso ergogénico, cafeína, performance.

\section{INTRODUÇÃO}

A cafeína é consumida regularmente por bilhões de pessoas no mundo, sendo vital para a economia de alguns países. A cafeína pertence ao grupo de compostos das metilxantinas e sua composição química principal é 1,3,7-trimetilxantina. Ela faz parte do grupo de bases da purina. A purina em si não ocorre na natureza, mas inúmeros derivados são biologicamente significativos. As bases deste grupo, que tem importância farmacêutica, são todos os derivados metilados da 2,6-dioxipurina (xantina). As xantinas são substâncias capazes de estimular o sistema nervoso, produzindo um certo estado de alerta de curta duração (SILVA, 2003).

Pode-se dizer que $80 \%$ da população geral faz uso dessa substância diariamente, embora seja muito difícil quantificar seu consumo (STRAIN \& GRIFFITHS, 2000). Devido a toda essa demanda foram realizadas diversas pesquisas em torno dos efeitos fisiológicos causados pela substância.

A cafeína afeta quase todos os sistemas do organismo, sendo que seus efeitos mais óbvios ocorrem no sistema nervoso central (SNC). Quando consumida em baixas dosagens $(2 \mathrm{mg} / \mathrm{kg})$, a cafeína provoca aumento do estado de vigília, diminuição da sonolência, alívio da fadiga, aumento da respiração, aumento na liberação de catecolaminas, aumento da freqüência cardíaca, aumento no metabolismo e diurese. Em altas dosagens $(15 \mathrm{mg} / \mathrm{kg}$ ) causa nervosismo, insônia, tremores e desidratação (CONLEE, 1991).

No meio esportivo, a cafeína tem sido freqüentemente utilizada por atletas na busca de benefícios ergogênicos que possam melhorar o rendimento em seus respectivos esportes (DANTAS, 2003).

A cafeína reduz o limiar de excitabilidade e prolonga a duração do período ativo da contração muscular, in vitro, por aumentar a liberação de cálcio do retículo sarcoplasmático para o sarcoplasma e por inibir o mecanismo de recaptação de cálcio pelo retículo sarcoplasmático, tornando o íon $\mathrm{Ca}^{+}$mais disponível para a contração muscular. No entanto, esse mecanismo de ação só pôde ser detectado em experimentos in vitro, utilizando-se dosagens muito altas de cafeína, cujas concentrações sanguíneas representam efeitos tóxicos para o organismo. Dadas essas con- dições, não é possível que a mobilização intracelular de cálcio do retículo sarcoplasmático represente um mecanismo nos efeitos ergogênicos da cafeína.

mecanismo mais favorável para explicar os efeitos ergogênicos da cafeína é através de sua função de antagonista dos receptores de adenosina e, portanto, ao impedir sua interação com adenosina, aumenta os níveis de AMPc (Adenosina Monofosfato Cíclica), provocando uma série de respostas no organismo, como: liberação de catecolaminas, aumento da pressão sanguínea, lipólise, aumento das secreções gástricas, aumento da diurese e ativação do sistema nervoso central (NEHLIG \& DEBRY, 1994; SAWYNOK \& YAKSH, 1993; TARNOPOLSKY, 1994).

Além deste mecanismo de ação, a cafeína exerce um efeito sobre a atividade da bomba $\mathrm{Na}+-\mathrm{K}+$. A cafeína influencia na regulação das concentrações de $\mathrm{K}+$ no meio extracelular e intracelular, mantendo as concentrações altas no meio intracelular e baixas no meio extracelular, o que contribui para o retardamento da fadiga. Tendo em vista que baixas concentrações de $K+$ no plasma ajudam a manter a excitabilidade das membranas celulares, nos músculos contráteis, observa-se que este pode ser outro mecanismo de ação a nível celular, capaz de explicar os efeitos ergogênicos da cafeína (LINDINGER et al., 1993).

Em uma das primeiras pesquisas realizadas, os avaliados executaram uma atividade física em bicicleta ergométrica, a $80 \%$ $\mathrm{VO}_{2}$ máx, até a exaustão, 1 hora após a ingestão de $330 \mathrm{mg}$ de cafeína. Os avaliados apresentaram um aumento de 19,5\% no tempo de endurance (COSTILL et al., 1978). Outra pesquisa identificou aumento na performance de $7 \%$ na quantidade de trabalho produzido em $2 \mathrm{~h}$ de exercício em bicicleta isocinética, com ingestão de $250 \mathrm{mg}$ de cafeína, porém aliada ao carboidrato (IVY et al., 1979). Outro estudo comprovou que exercício de bicicleta ergométrica a 65-70\% $\mathrm{VO}_{2}$ máx, durante 30 minutos, com uma ingestão de $5 \mathrm{mg} / \mathrm{kg}$ de cafeína, resultou numa economia de $42 \%$ do glicogênio muscular (ESSING et al., 1980).

Alguns estudos apontam para um relativo aumento da força muscular, acompanhado de uma maior resistência à aparição da fadiga muscular após a ingesta de doses relativamente altas 
Tabela 1 - Dados referente aos sujeitos da pesquisa

\begin{tabular}{|c|c|c|c|c|c|c|c|c|c|c|}
\hline & $\mathrm{N}$ & $X$ & Med & $S$ & Min & Max & Curto-se & Resul. & Asimetría & Resul. \\
\hline Edad (año) & 12 & 33,5 & 31 & 8,0 & 20 & 49 & $-0,04$ & Plati_Curt & 0,37 & asimétrico \\
\hline Altura $(\mathrm{cm})$. & & 176,1 & 176 & 7,8 & 162,5 & 188,5 & $-0,85$ & & $-0,04$ & \\
\hline Peso $(\mathrm{Kg})$ & & 69 & 68,2 & 8,4 & 55,2 & 82,3 & $-0,62$ & & 0,18 & \\
\hline Tiempo de formación (año) & & 12,8 & 15 & 7,1 & 1 & 21 & $-1,36$ & & $-0,40$ & \\
\hline
\end{tabular}

de cafeína (KALMAR \& CAFARELLI, 1999; LOPES, 1983; PINTO \& TARNOPOLSKY,1997; ROY,1994). Embora não se saiba de forma concreta o verdadeiro mecanismo de ação responsável pelo aumento da força muscular. Todavia, acredita-se que isso ocorra em maior intensidade muito mais pela ação direta da cafeína no SNC, do que pela sua ação em nível periférico (KALMAR \& CAFARELLI, 1999).

Em uma pesquisa utilizando a dosagem de $9 \mathrm{mg} / \mathrm{kg}$ de cafeína, foi relatado o aumento no tempo de endurance na corrida e no ciclismo de $44 \%$ e $51 \%$, respectivamente (GRAHAM et al., 1991).

Porém, alguns anos depois, foi identificado que esta dosagem de $9 \mathrm{mg} / \mathrm{kg}$ de cafeína já caracterizava concentrações urinárias acima do estabelecido pelo $\mathrm{COI}$, sendo considerada dopping (PASMAN et al., 1995); o Comitê Olímpico Internacional classifica a cafeína como uma droga restrita, positiva em concentrações acima de $12 \mu \mathrm{g} / \mathrm{ml}$ na urina.

Desde de janeiro de 2004, a cafeína encontra-se no programa de monitoramento da WADA (World Anti-Doping Agency), não sendo considerada substância proibida, mas sendo somente analisada por ocasião de competições (WORLD ANTI-DOPING AGENCY, 2004).

Na literatura científica analisada, poucos estudos foram encontrados em relação ao treinamento de força, de alta intensidade, velocidade e potência. Os estudos analisados refletem uma controvérsia muito grande em relação ao tema em questão.

Com relação aos exercícios máximos e supramáximos de curta duração, a maioria dos estudos dessa natureza vem demonstrando que a ingestão de cafeína pode melhorar significativamente $o$ desempenho físico em exercícios máximos de curta duração (até 5 minutos) (GRAHAM et al., 1998).

A Potência Aeróbica Máxima de um atleta é a quantidade de energia que ele utiliza a cada minuto, quando o seu consumo de $\mathrm{O}_{2}$ é igual ao seu $\mathrm{VO}_{2}$ máx. Em geral, a PAM não consegue ser sustentada por mais de 7 minutos ininterruptos, isto é, quanto

Gráfico 1 - Percentuais médios da Potência Aeróbica Máxima capazes de ser sustentados em função da duração da prova

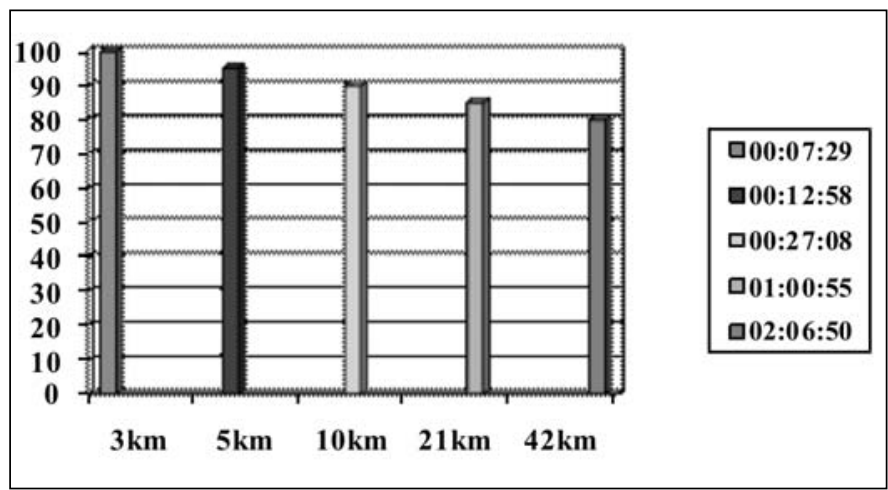

maior for a duração da prova, menor será a \%PAM utilizada (Gráfico 1).

Foi observada a existência de alguns trabalhos de pesquisa em torno do efeito da cafeína no retardamento da fadiga muscular, avaliando-se unicamente os tempos máximos de exercícios, com intensidades submáximas. Dessa forma, o objetivo do presente estudo é verificar se o efeito se dá, também, em tempos de corridas entre 5 e 10 minutos, com intensidades máximas.

\section{OBJETIVO}

Esta pesquisa tem como objetivo verificar os efeitos ergogênicos da cafeína em teste máximo de 3.200 metros.

\section{MATERIAIS E MÉTODOS}

\section{Amostra}

A amostra do presente estudo foi composta por 12 atletas, do sexo masculino, de corrida de ruas, desde 5 a $42 \mathrm{~km}$, com idade média de 33,5 \pm 8 anos, estatura de 176,1 $\pm 7,8 \mathrm{~cm}$, peso corporal de $68,9 \pm 8 \mathrm{~kg}$ e tempos de treinamento de $13 \pm 7,3$ anos, conforme apresentado na Tabela 1 e no Gráfico 2.

Havia mais três sujeitos, uma mulher e dois homens, que foram retirados da amostra por terem sofrido forte indisposição gástrica antes e durante o teste, por efeito da sensibilidade à cafeína.

Todos declararam ser não fumantes, não consumidores de bebida alcoólica, apenas 2 dos sujeitos informaram ser consumidores de cerveja 1 vez na semana ou 1 vez no mês. Declararam, ainda, não consumir medicamentos à base de cafeína, tendo sido verificado o uso de anti-inflamatórios, complexos vitamínicos, sulfato ferroso e supradin, de uma forma geral entre os atletas.

Nenhum dos avaliados declarou estar sofrendo de lesão muscular ou outra lesão que impeça a realização dos testes na potencialidade máxima de cada atleta. Os avaliados tomaram conhecimento de todos os procedimentos do teste, através do termo de consentimento, o qual foi lido e assinado por todos.

\section{Gráfico 2 - Característica da amostra}

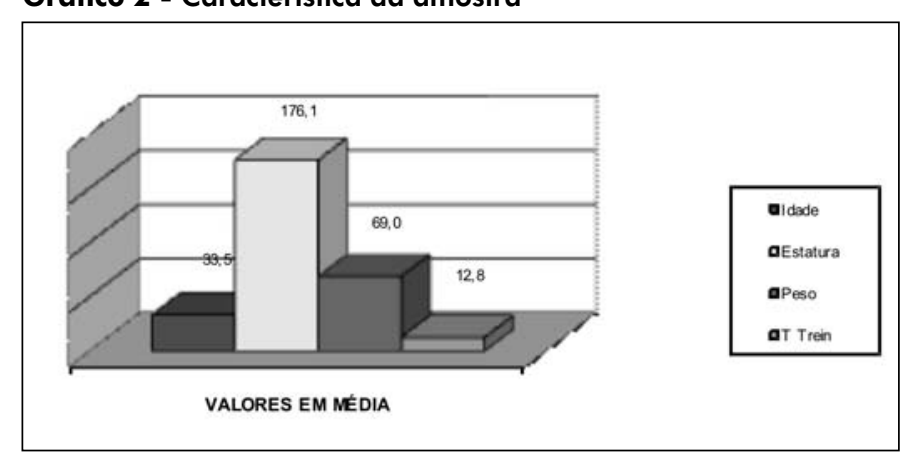




\section{Procedimentos Éticos}

Este trabalho foi realizado em conformidade com as "Normas de Realização de Pesquisa em Seres Humanos", Resolução n' 196/96 do Conselho Nacional de Saúde, de 10/10/96 (BRASIL, 1996). Ressalta-se que esta pesquisa foi submetida a análise do COMEP - Comitê de Ética em Pesquisa, envolvendo Seres Humanos, da Universidade Castelo Branco - RJ, tendo sido emitido parecer favorável.

\section{Procedimentos da Pesquisa}

Foram realizados dois testes máximos de 3.200 metros, no mesmo horário, em dias distintos. No primeiro dia foram realizadas as avaliações da estatura e do peso corporal, a entrevista e, em seguida, o teste. O intervalo entre os testes foi de uma semana, durante a qual houve uma abstinência de cafeína. No $2^{\circ}$ dia de teste, foi realizada a ingestão da cafeína e aguardado o prazo de 1 hora para o início do teste, até que essa substância fosse metabolizada.

\section{PROCEDIMENTOS PRELIMINARES}

\section{Controle Nutricional}

Todos declararam que, durante o período de intervalo entre os testes, mantiveram a abstinência de qualquer alimento com cafeína, dentre eles o chocolate e produtos à base de cacau, açaí, guaraná em pó, porangaba, chás pretos (mate, iced tea, bebidas energéticas), refrigerantes à base de cola e de guaraná e o próprio café.

Foi realizada a abstinência da referida substância neste estudo devido ao fato de que, em consumidores habituais de $200 \mathrm{mg} /$ dia de cafeína, foi ministrado $6 \mathrm{mg} / \mathrm{kg}$ da referida substância 1 hora antes de uma corrida de 90 minutos na esteira a $70 \% \mathrm{VO}_{2}$ máx, e não foi identificado efeito ergogênico, concluindo que a habituação da cafeína neutraliza as respostas metabólicas, eliminando seus efeitos ergogênicos (TARNOPOLSKY et al., 1989).

\section{Monitoramento da Condição Climática}

Foi utilizado o termohigrômetro TZ-18 (INCOTERM, Brasil) para serem aferidas a temperatura e a umidade relativa do ar (URA)

\section{Gráfico 3 - Tempos de corrida observados}

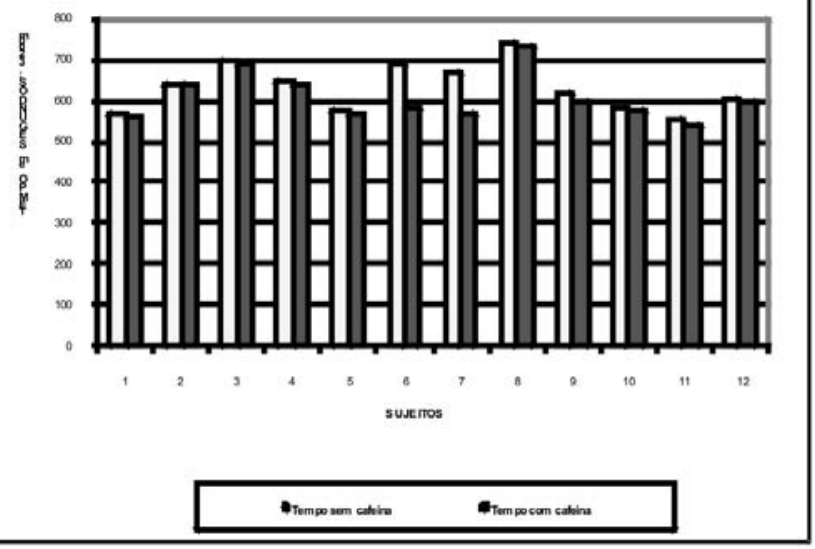

antes de cada um dos testes. $O$ primeiro teste foi realizado com uma temperatura de $29,1^{\circ}$ e URA de $64 \%$. O segundo teste foi realizado com uma temperatura de $27^{\circ}$ e URA de $71 \%$.

\section{Avaliação do Peso Corporal e Estafura}

A referida avaliação foi realizada com o auxílio de uma balança eletrônica personal line com estadiômetro e com calibragem automática (FILIZOLA, Brasil) no ato do seu funcionamento. A avaliação do peso corporal e da estatura foi realizada entre 6 h30min e 7 h30min da manhã, e os sujeitos vestiam apenas um short de corrida.

\section{Avaliação do $V \boldsymbol{O}_{2} \mathbf{m a ́ x}$}

Foi utilizado o protocolo do teste de corrida de 3.200 metros de RIBILS \& KACHODORIAN (apud FERNANDES FILHO, 2003), válido para a avaliação de grupos de indivíduos com amplo nível de aptidão física e de faixa etária bem variável, pois na fórmula de cálculo de $\mathrm{VO}_{2}$ máx são levados em consideração o tempo gasto na realização da tarefa $(X 1)$, em segundos, a idade (X2), em anos, e o peso corporal (X3), em Kg, sendo expressa nos seguintes termos:

$$
\begin{gathered}
\mathrm{VO}_{2} \text { máx }\left(\mathrm{ml} \cdot \mathrm{kg}^{-1} \cdot \mathrm{min}^{-1}\right)=114,496-0,04689(X 1)-0,37817- \\
(\mathrm{X} 2)-0,15406(X 3)
\end{gathered}
$$

\section{Moniforamenfo do Treinamento}

Na semana entre testes, os treinamentos da equipe foram realizados normalmente como previsto pelo seu técnico, computando um volume total de $84,4 \mathrm{Km}$ semanais pela manhã (entre Long Slow Distance, intervalados e corridas contínuas) e $21 \mathrm{Km}$ semanais pela tarde (corridas contínuas).

\section{Administração da Cafeína}

A cafeína foi administrada aos atletas, no segundo dia de testes, na quantidade de $5 \mathrm{mg}$ por quilo de peso corporal, sob a forma de cápsulas, confeccionadas, precisamente nos valores acima descritos, em farmácia de manipulação.

\section{Gráfico $4-\mathrm{VO}_{2 \text { máx }}$ de cada sujeito nos dois testes}

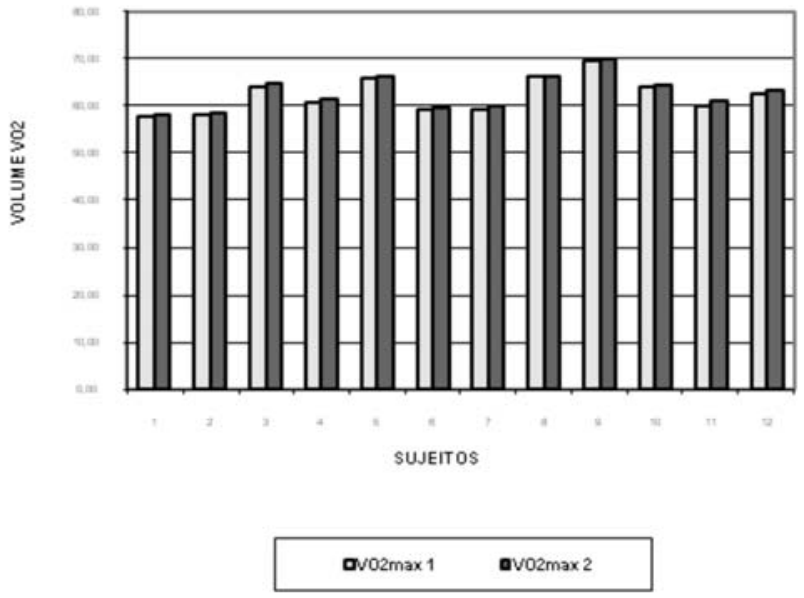


Tabela 2 - Teste de 3.200 metros sem cafeína

\begin{tabular}{lccc}
\hline & $\mathrm{N}$ & $\mathrm{X}$ & $\mathrm{s}$ \\
\hline $\mathrm{VO}_{2 \max } 1$ & 12 & 62,28 & 4,22 \\
$\mathrm{~T}$ (seg.)1 & 12 & 616,92 & 56,8 \\
\hline
\end{tabular}

Tabela 4 - Estatística inferencial

\begin{tabular}{lcccc}
\hline $\mathrm{n}=12$ (homens) & $\mathrm{t}$ & $\mathrm{gl}$ & $\mathrm{r}$ & Sig*. \\
\hline $\mathrm{VO}_{2 \max } 1 / \mathrm{VO}_{2 \max } 2$ & $-4,660$ & 11 & 0,998 & 0,00069 \\
$\mathrm{~T}$. (seg.)1 / T. (seg.)2 & 2,171 & 11 & 0,902 & 0,00069 \\
\hline
\end{tabular}

$p<0,05$

A administração se deu por meio de cápsulas (pura), já que, assim, a substância exerce um maior potencial ergogênico (GRAHAM et al., 1998).

Em média, cada atleta consumiu $344,8 \mathrm{mg}$ da substância analisada, o que equivale a uma caneca e meia $(280 \mathrm{ml})$ de café. Juntamente com a cafeína, foi ingerida a quantidade média de $200 \mathrm{ml}$ de Gatorade.

Optou-se por administrar a quantidade de $5 \mathrm{mg}$ por quilo de peso corporal por ter sido verificado ser unânime em todos os artigos científicos analisados que esta é uma quantidade confirmadamente afastada do risco de dopping $(9 \mathrm{mg} / \mathrm{kg})$, porém com comprovado efeito ergogênico. Quando ingerida em dosagens de aproximadamente $5 \mathrm{mg} / \mathrm{kg}$, $1 \mathrm{~h}$ antes do exercício, a cafeína exerce benefícios ergogênicos sem atingir valores na concentração urinária considerados dopping (BRAGA \& ALVES, 2000).

Não foram constatadas diferenças significativas entre as dosagens de 5, 9 e $13 \mathrm{mg} / \mathrm{Kg}$. Porém, as duas últimas ultrapassaram as concentrações limites definidas pelo $\mathrm{COI}$ (Comitê Olímpico Internacional) como dopping (12mg/l de urina) (PASMAN et al., 1995).

\section{APRESENTAÇÃO E DISCUSSÃO DOS DADOS}

Com os dados obtidos durante os testes pode-se observar o $\mathrm{VO}_{2 \max }$ e o tempo em segundos do primeiro teste, sem a utilização de cafeína (Tabela 2) e do segundo teste, com a ingestão da cafeína (Tabela 3).

O resultado do grupo amostral do teste de 3.200 metros sem administração de cafeína apresentou um valor de $\mathrm{x}=616,92$ $+56,8$ segundos.

O resultado do grupo amostral do teste de 3.200 metros com administração de cafeína apresentou um valor de $\mathrm{x}=608,83$ $+58,22$ segundos.

Observando os dados obtidos, percebe-se uma diferença na média do tempo e do $\mathrm{VO}_{2 \max }$ entre os dois testes. $\mathrm{O}$ Gráfico 3 detalha melhor a diferença nos tempos de cada sujeito e o Gráfico 4, o $\mathrm{VO}_{2 \max }$

Pode-se observar que, com a exceção do atleta 02, todos os atletas tiveram uma melhora nos tempos entre o primeiro teste (sem cafeína) e o segundo teste (com cafeína).

Pode-se observar que todos os atletas tiveram uma melhora no $\mathrm{VO}_{2 \max }$ entre o primeiro teste (sem cafeína) e o segundo teste (com cafeína).
Tabela 3 - Teste de 3.200 metros com cafeína

\begin{tabular}{lccc}
\hline & $\mathrm{N}$ & $\mathrm{X}$ & $\mathrm{s}$ \\
\hline $\mathrm{VO}_{2 \text { max }} 2$ & 12 & 62,66 & 4,27 \\
$\mathrm{~T}$. (seg.)2 & 12 & 608,83 & 58,22 \\
\hline
\end{tabular}

Tabela 5 - Variáveis comparativas entre tempos e $\mathrm{VO}_{2}$ máx

\begin{tabular}{lc}
\hline$n=12$ (homens) & $M \pm S D$ \\
\hline$\Delta \mathrm{T}$ & $8,08 \pm 6,01 \mathrm{seg}$ \\
$\Delta \mathrm{T} \%$ & $1,33 \pm 1,01 \%$ \\
$\Delta \mathrm{VO}_{2}$ & $0,38 \pm 0,28 \mathrm{ml} / \mathrm{kg} / \mathrm{min}$ \\
$\Delta \mathrm{VO}_{2} \%$ & $0,61 \pm 0,46 \%$ \\
\hline
\end{tabular}

No tratamento estatístico dos dados obtidos, foi realizada uma estatística inferencial verificando coeficiente de correlação " $r$ ", o teste " $t$ " pareado com grau de liberdade (gl) 11 e uma significância de $p<0,05$ (Tabela 4).

Foi verificado que 11 entre 12 atletas obtiveram uma melhora nos tempos médios de performance no teste de corrida máxima, na distância de 3.200m (Gráfico 3), da ordem de 0,13 \pm 0,1 $\min (8,08 \pm 6,01 \mathrm{seg})$, tendo o grupo realizado no primeiro teste o tempo de 10,28 \pm 0,95 min e no segundo 10,15 \pm 0,97, apresentando 1,33 $\pm 1,01 \%$ de redução no tempo de realização do teste (Tabela 5).

Porém, ao se analisar o $\mathrm{VO}_{2}$ máx (Gráfico 4), verifica-se que houve um ganho de 0,38 $\pm 0,28 \mathrm{ml} / \mathrm{kg} / \mathrm{min}$, resultando em uma melhora de 0,61 0,46\%. Esta menor variação do $\mathrm{VO}_{2}$. máx em relação ao tempo de prova, deve-se, provavelmente, à diferença apresentada na umidade relativa do ar que variou em 7 pontos \%. O aumento significativo da URA no $2^{\circ}$ teste $(71 \%)$ em relação ao $1^{\circ}(64 \%)$, pode explicar a variação no $\mathrm{VO}_{2}$ máx não proporcional à melhora no tempo médio dos atletas. A temperatura no $1^{\circ}$ teste foi de $29,1^{\circ} \mathrm{C}$ e no segundo foi de $27^{\circ} \mathrm{C}$, não caracterizando uma diferença que possa ser considerada interveniente na avaliação.

Três entre quinze atletas apresentaram desconforto gástrico, sudorese, tonteira e, até mesmo, ânsia de vômito após a ingestão da cafeína, tendo sido eliminados da amostra. Já havia sido observada a existência de alguns efeitos colaterais, nesses níveis de dosagens (DANTAS, 2003).

\section{CONCLUSÃO}

Observou-se uma melhora do tempo médio de performance com o uso de cafeína, após 1 semana de abstinência desta substância, em 11 dos 12 sujeitos avaliados, da ordem de 8 segundos, bastante relevante em se tratando de testes máximos.

Houve uma melhora significativa entre os dois testes de 3.200 metros realizados, possibilitando a aceitação da influência da cafeína na performance dos sujeitos.

Dessa forma, concluímos que o uso da cafeína, após uma semana de abstinência, produz efeito ergogênico significativo na performance de atletas em provas de meio-fundo, isto é, para tempos médios entre 5 e 10 minutos. Para futuros estudos, ressaltamos a importância da avaliação em testes de corrida com duração acima de 60 minutos. 


\section{BIBLIOGRAFIA}

BELL, D.G.; MCLELLAN, T.M.; SABISTON, C.M. Effect of ingesting caffeine and ephedrine on 10-km run performance. Medicine-and-Science-in-Sports-and-Exercise. 34 (2): 344-349, 2002.

BRAGA, L.C. \& ALVES, M.P. A cafeína como recurso ergogênico nos exercícios de endurance. Rev. Bras. Ciên. E Mov., 8(3): p. 33-37, 2000.

CONLEE, R.K. Amphetamine, caffeine and cocaine. In: D.R. LAMB, M.H. WILLIAMS (Eds) Ergogenics: Enhancement of Performance in Exercise and Sport. New York, Benchmark Press, p. 285-310, 1991.

CONWAY, K.J.; ORR, R. \& STANNARD, S.R. Effect of a divided caffeine dose on endurance cycling performance, postexercise urinary caffeine concentration, and plasma paraxanthine. Journal-of-Applied-Physiology. 94 (4): 1557-1562, 2003.

COSTILL, D.L.; DALSKY, G.P. \& FINK, W.J. Effects of caffeine on metabolism and exercise performance. Med. Sci. Sports Exerc., 10(3): p.155-158, 1978.

DANTAS, E.H.M. A Prática da Preparação Física. 5 ed. Rio de Janeiro: Shape, 2003.

GRAHAM, T.E.; HIBBERT, E. \& SATHASIVAM, P. Metabolic and exercise endurance effects of coffee and caffeine ingestion. J. Appl. Physiol. 85(3): 883-889, 1998.

ESSING, D.; COSTILL, D.L. \& VAN HANDEL, P.J. Effects of caffeine ingestion on utilization of muscle glycogen and lipid during leg ergometer cycling. Med. Sci. Sports Exerc. 1 , p.86-90, 1980.

FERNANDES FILHO, J. A Prática da Avaliação Física. 2 ed. Rio de Janeiro: Shape, 2003. GRAHAM, T.E.; \& SPRIET, L.L. Metabolic, catecholamine and exercise performance to varying doses of caffeine. J. Appl. Physiol.,78(3): p.867-874, 1995.

GRAHAM, T.E. \& SPRIET, L.L. Performance and metabolic responses to a high caffeine dose during prolonged exercise. J. Appl. Physiol., 71: p.2292-2298, 1991.

GRAHAM, T.E.; HIBBERT, E. e SATHASIVAM, P. Metabolic and exercise endurance effects of coffee and caffeine ingestion. J. Appl. Physiol. 85(3): 883-889, 1998.

IVY, J.L.; COSTILL, D.L.; FINK, W.J. \& LOWER, R.W. Influence of caffeine and carbohidrate feedings on endurance performance. Med. Sci. Sports Exerc., 11(1): p.6-11, 1979.

KALMAR, J.M. \& CAFARELLI, E. Effects of caffeine on neuromuscular function. J. Appl. Physiol., 87 (2): p.801-808, 1999.
LINDINGER, M.I.; GRAHAM, T.E. e SPRIET, L. Caffeine atenuates the exercise-induced increase in plasma [K+] in humans. J. Appl. Physiol. 74(3): 1149-1155, 1993.

LOPES, J.M. et al. Effect of caffeine on skeletal muscle function before and after fatigue. J. Appl. Physiol., 54 (5): p.1303-1305, 1983.

NEHLIG, A. \& DEBRY, G. Caffeine and sports activity: a review. Int. J. Sports Med. 15: 215-223, 1994.

PALUSKA, S.A. Caffeine and exercise. Curr-Sports-Med-Rep. 2(4): 213-219, 2003.

PASMAN, W.J.; BAAK, M.A.; JEUKENDRUP, A.E. \& HAAN, A. The effect of different dosages of caffeine on endurance performance time. Int. J. Sports Med., 16(4): p.225-230, 1995.

PINTO, S. \& TARNOPOLSKY, M. Neuromuscular effects of caffeine in males and females. Can. J. Appl.Physiol., 22: p.48, 1997.

ROY, B. et al. Caffeine and neuromuscular fatigue in endurance athletes. Can. J. Appl. Physiol., 19: p.41, 1994

SAWYNOK, J. e YAKSH, T.L. Caffeine as an analgesic adjuvant: a review of pharmacology and mecanisms of action. Pharmalogical Reviews. 45(1): 43-51, 1993.

SILVA, M. S. Os efeitos da cafeína relacionados à atividade física: uma revisão. Efdeportes; Ano 9 - no 66 - novembro de 2003.

SPRIET, L.L. et al. Caffeine ingestion and muscle metabolism during prolonged exercise in humans. Am. J. Physiol. 262: E891-E898,1992.

SUTTON, J.R. Physiological responses to caffeine during endurance running in habitual caffeine users. Med. Sci. Sports Exerc., 21 (4): p.418-424, 1989

TARNOPOLSKY, M.A. Caffeine and endurance performance. Sports Med. 18 (2): 109-125, 1994.

et al. Physiological responses to caffeine during endurance running in habitual caffeine users. Med. Sci. Sports Exerc. 21(4):

Protein, caffeine and sports. The Physician Sports Med. 21 (3): 137-149, 1993.

et al. Caffeine related disorders. In: SADOCK, B.J.; SADOCK, V.A. Kaplan \& Sadock's Comprehensive Textbook of Psychiatry. Baltimore, Lippincott, 7th, p. 982-90, 2000.

WORLD ANTI-DOPPING AGENGY. 2004 Monitoring Program. Disponível em: <http://www wada-ama.org/en/†l.asp>. Acesso em: 29 jun. 2004 